\title{
Transcription Initiation Factor TFIID Subunit 3
}

National Cancer Institute

\section{Source}

National Cancer Institute. Transcription Initiation Factor T FIID Subunit 3. NCI Thesaurus.

Code C30064.

Transcription initiation factor TFIID subunit 3 (929 aa, $104 \mathrm{kDa}$ ) is encoded by the human TAF3 gene. This protein plays a role in both the regulation of transcription and myoblast differentiation. 